\title{
MHD Supernova Jets: The Missing Link
}

\author{
David L. Meier and Masanori Nakamura \\ Jet Propulsion Laboratory, California Institute of Technology, \\ Pasadena, CA 91109
}

\begin{abstract}
We review recent progress in the theory of jet production, with particular emphasis on the possibility of 1) powerful jets being produced in the first few seconds after collapse of a supernova core and 2) those jets being responsible for the asymmetric explosion itself. The presently favored jet-production mechanism is an electrodynamic one, in which charged plasma is accelerated by electric fields that are generated by a rotating magnetic field anchored in the protopulsar. Recent observations of Galactic jet sources provide important clues to how all such sources may be related, both in the physical mechanism that drives the jet in the astrophysical mechanisms that create conditions conducive to jet formation. We propose a grand evolutionary scheme that attempts to unify these sources on this basis, with MHD supernovae providing the missing link. We also discuss several important issues that must be resolved before this (or another scheme) can be adopted.
\end{abstract}

\subsection{Introduction: A Cosmic Zoo of Galactic Jet Sources}

The last few decades have seen the discovery of a large number of different types of Galactic sources that produce jets. The purpose of this talk is to show that all of these jets sources are related, in both a physical sense and an astrophysical sense. Furthermore, Craig Wheeler's idea that most core collapse supernovae (SNe) are driven by MHD jets from a protopulsar provides the missing link in an attractive unified scheme of all stellar jet sources. Below is a list of Galactic jet sources that have been identified so far:

(1) Jets from Stars Being Born: Protoplanetary systems. These are jetted and bi-polar outflows from young stars or star-forming regions. They are associated with protostars that have protoplanetary disks and are ejected at approximately the escape velocity of the central star $\left(v_{\text {jet }} \sim v_{\text {esc }} \sim 200 \mathrm{~km} \mathrm{~s}^{-1}\right)$.

(2) Jets from Dying Stars:

- Planetary Nebula Systems. Stars with initial mass less than a few $M_{\odot}$ end their lives as a planetary nebula, leaving a white dwarf remnant. Many PN have bipolar shapes; some even have highly-collimated outflows of up to $v_{\text {jet }} \sim 1000 \mathrm{~km} \mathrm{~s}^{-1}$, the escape speed from the surface of a rather distended white dwarf.

- Core-collapse Supernovae: Stars with masses between roughly 10 and $30 M_{\odot}$ are destroyed in an explosion that is triggered by the collapse of their iron cores. They leave a neutron star/pulsar remnant. There is growing evidence (see below) that corecollapse $\mathrm{SNe}$ also produce jets with a power comparable to the explosion itself. Ex- 
pected speeds are $v_{\text {jet }} \sim 0.25-0.5 c-$ the escape speed from the new protoneutron star.

- Isolated young pulsars (Crab, Vela, etc.): Jets now have been detected by Chandra in these objects and have speeds $v_{j e t} \sim 0.5 c$. These may be the remaining ghosts of core-collapse jets that occurred thousands of years ago.

- Gamma-ray bursts (GRBs): Believed to be black holes in formation, these produce jets with $\Gamma_{j e t} \sim 100-300$ that point toward us. Long-duration GRBs are closely associated with powerful SNe and may represent the death of particularly massive $\left(>30 M_{\odot}\right.$ ) stars.

(3) Jets from Re-kindled Dead Stars in Binary Systems

- Symbiotic stars (e.g., R Aquarii): These tend to be accreting white dwarfs where the companion star has recently left the main sequence and expanded into a red giant, transferring mass to the compact object via a strong wind or Roche lobe overflow. Jets are produced by the white dwarf and have speeds up to $v_{\text {jet }} \sim 6000 \mathrm{~km} \mathrm{~s}^{-1}$, the escape speed from a white dwarf.

- Neutron star X-ray Binaries: These occur in both low-mass and high-mass systems. Jets tend to appear at lower accretion rates. Typical speeds are $\sim 0.25 c$, but can reach speeds approaching $c$.

- SS433-type objects: Observed properties suggest a super-Eddington accreting, magnetized neutron star in a high-mass binary system. Jets have speed $v_{\text {jet }} \sim 0.25 c$.

- Classical microquasars (GRS 1915+105, GRO J1655-40, GX 339-4, etc.): These produce jets with $v_{j e t}>0.6-0.95 c\left(\Gamma_{j e t} \equiv\left[1-v_{j e t}^{2} / c^{2}\right]^{-1 / 2}>1.25-3\right)$ and up. Virtually all are black hole candidates in low-mass X-ray binary systems.

The inclusion of core-collapse $\mathrm{SNe}$ above is the key to the unified model presented below. In the mid 1990s it was discovered that such SNe emit polarized light in the optical band, caused by electron scattering by an asymmetrically-expanding explosion |Wang et al. 2001. Wang et al. 2003 Leonard et al. 2001|. The variation of polarization properties with time and with different $\mathrm{SN}$ types gives important clues to its nature. In a given $\mathrm{SN}$, the degree of polarization $\Pi$ increases with time but the polarization direction remains constant in time and wavelength, indicating that the asymmetry is global and maintains a fixed direction. For Type IIa SN (ones with a large hydrogen-rich envelope), the $\Pi \sim 1 \%$, indicating only a 2:1 or less asymmetry. For Type IIb SN (ones that have lost their hydrogen envelope prior to the explosion, leaving only the helium envelope), $\Pi$ is higher $(\sim 2 \%)$, indicating a 2.5:1 axial ratio. For SN Type Ib/Ic (ones that have lost most or all of their envelope, leaving a compact blue Wolf-Rayet star that then explodes), $\Pi$ is quite high (4-7\%), indicating a 3:1 axial ratio or better. Clearly, the deeper one sees into the explosion, the more elongated the exploding object appears. These observers have concluded that core-collapse SN have a global prolate shape that appears to be associated with the central engine producing the explosion. A jet, with energy comparable to that of the explosion itself, significantly alters the shape of the envelope, creating the elongated, polarized central source. In the paper below we will show that all of the above sources may be intimately related, both in the physical origins of the jet itself and in their astrophysical origins as well. 


\subsection{Basic Principles of Magnetohydrodynamic Jet Production}

The basic principles of MHD jet production have been described elsewhere [Meier et al. 2001].

The reader is referred to that paper for a more detailed description, and more comprehensive figures, than in the short review given below.

\subsubsection{Launching of the Jet Outflow: The Jet Engine Itself}

\subsubsection{Jet Production in Accreting Systems and Pulsars}

Several mechanisms for producing bipolar outflows have been suggested (explosions in the center of a rotationally-flattened cloud, radiation-pressure-driven outflows from a disk, etc.), but none of these is able to produce outflows approaching the highly-relativistic speeds observed in the fastest jet sources. The currently-favored mechanism is an electromagneto-hydrodynamic (EMHD) one, somewhat similar to terrestrial accelerators of particle beams. Indeed, electromagnetic acceleration of relativistic pulsar winds has been a leading model for these objects since the 1960s. EMHD jet production was first suggested in 1976 [Blandford 1976] Lovelace 1976] and has been applied model to rotating black holes [Blandford \& Znajek 1977] (BZ) and to magnetized accretion disks [Blandford \& Payne 1982] (BP). This mechanism has now been simulated and is sometimes called the "sweeping pinch" mechanism [Shibata \& Uchida 1985, Kudoh et al. 1999, Nakamura et al. 2001].

The most important ingredient in the EMHD mechanism is a magnetic field that is anchored in a rotating object and extends to large distances where the rotational speed of the field is considerably slower. Plasma trapped in the magnetic field lines is subject to the Lorentz $(J \times B)$ force, which, under conditions of high conductivity (the MHD assumption), splits into two vector components: a magnetic pressure gradient $\left(-\nabla B^{2} / 8 \pi\right)$ and a magnetic tension $(B \cdot \nabla B / 4 \pi)$. Differential rotation between the inner and outer regions winds up the field, creating a strong toroidal component ( $B_{\phi}$ in cylindrical $[R, Z, \phi]$ coordinates). The magnetic pressure gradient up the rotation axis $\left(-d B_{\phi}^{2} / d Z\right)$ accelerates plasma up and out of the system while the magnetic tension or "hoop" stress $\left(-B_{\phi}^{2} / R\right)$ pinches and collimates the outflow into a jet along the rotation axis.

This basic configuration of differential rotation and twisted magnetic field accelerating a collimated wind can be achieved in all objects identified in Sect. 1.1 For protostars, white dwarfs, X-ray binaries, classical microquasars and GRBs, the field will be anchored in the accreting plasma, which may lie in a rotating disk (BP) and/or may be trapped in the rotating spacetime of the spinning central black hole itself (BZ). In the case of SS433-type objects, isolated pulsars, and core-collapse SN the rotating field is anchored in the pulsar (or protopulsar). In SS433 and core-collapse SN the source of the accelerated plasma is, once again, accretion, but in isolated pulsars it is believed to be particles created in spark gaps by the high $\left(10^{12} \mathrm{G}\right)$ field.

\subsubsection{Jets from Kerr Black Holes: Direct \& Indirect Magnetic Coupling}

The jet-production mechanism envisioned by BZ generally involved direct magnetic coupling of the accelerated plasma to the rotating horizon. That is, magnetic field lines thread the horizon, and angular momentum is transferred along those field lines to the external plasma via magnetic tension. However, another, indirect, coupling is possible. This mechanism, suggested by [Punsly \& Coroniti 1990] (PC) and recently simulated by us Koide et al. 2002], has the same effect as the BZ mechanism (extraction of angular mo- 
mentum from the rotating black hole by the magnetic field), but the field lines do not have to thread the horizon itself. Instead, they are anchored in the accreting plasma. When this plasma sinks into the ergosphere near the black hole $\left(R<2 G M / c^{2}\right)$, frame dragging causes the plasma to rotate with respect to the exterior, twisting up the field lines in a manner similar to the situation when the field is anchored in a disk or pulsar. (This occurs even if the accreting plasma has no angular momentum with respect to the rotating spacetime.) The twisted field lines then have two effects:

(1) Electromagnetic power is ejected along the rotation axis in the form of a torsional Alfven wave. Eventually the output Poynting flux power should be dissipated in the production and acceleration of particles and a fast jet.

(2) The back-reaction of the magnetic field accelerates the ergospheric plasma (in which it is anchored) to relativistic speeds against the rotation of the black hole. The counter-rotating ergospheric plasma now formally has negative angular momentum and negative energy (negative mass); that is, it has given up more than its rest mass in energy to the external environment. It is on orbits that must intersect the black hole horizon, and, when it does, the mass of the black hole decreases by a value equal to that negative energy.

This process is the magnetic equivalent of the Penrose process, but instead of extracting black hole rotational energy by particle scattering, the energy is extracted by scattering of an Alfven wave off the ergospheric plasma particles. Determining whether the BZ or PC process occurs in certain systems is an important question for future study.

\subsubsection{Acceleration and Collimation $(A \& C)$}

\subsubsection{Slow A \& C is Probably the Norm}

There are both theoretical and observational reasons for believing that slow acceleration and collimation is probably the norm for jet outflows in these sources. Nonrelativistic [Krasnopolsky et al. 1999] and relativistic [Vlahakis \& Konigl 2001] models of MHD wind outflows attain solutions where the wind opening angle is wide near the accretion disk and then narrows slowly over several orders of magnitude in distance from the disk. Because the dynamical time scale is of order $0.1 \mathrm{~ms}$ or less in these objects, a steady state is set up fairly quickly in jet ejection events that last even only a few seconds. In a steady state, the wind accelerates as it expands vertically away from the rotator. A jet is not fully formed until its speed exceeds the local wave propagation speed, i.e., the total Alfven speed $V_{A}=\left[\left(B_{R}^{2}+B_{Z}^{2}+B_{\phi}^{2}\right) /(4 \pi \rho)\right]^{1 / 2}$, where $\rho$ is the mass density in the outflowing material. The place where this occurs, often called the Alfven point or Alfven surface, generally is well above the rotating object producing the accelerating torsional Alfven wave. Analytic [Blandford \& Payne 1982 Li et al. 1992] and numerical [Krasnopolsky et al. 1999] studies of this steady state show that the outflow is rather broad at the base, and it slowly focuses as it is accelerated. At a height $Z_{A}>10 R_{0}$ above the disk, the total Alfven speed is exceeded, the flow is focused into a narrow cylindrical or conical flow, and little more acceleration and collimation takes place. The terminal jet speed $v_{j e t}$ is of order $V_{A}\left(Z_{A}\right)$, and this speed is usually of order the escape speed from the central rotator $V_{e s c}\left(R_{0}\right)$.

Furthermore, there now is observational evidence that, in at least some extragalactic systems, the steady-state picture of slow acceleration and collimation is correct. Very high resolution VLBA radio images of the M87 jet [Junor et al. 1999] show a broad 60 deg opening angle at the base that narrows to only a few degrees after a few hundred Schwarzschild 
radii. In addition, it has been argued [Sikora \& Madejski 2001] that most quasar jets must be broad at the base: they lack soft, Comptonized and relativistically-boosted X-ray emission that would be expected from a narrow, relativistic jet flow near the black hole.

\subsubsection{Stability of Highly-Magnetized Flows During A \& C}

Because the acceleration and collimation is expected to be slow, the terminal velocity and final state of the outflow will be reached only far from the central engine. The character of the outflow, therefore, will depend crucially on how it interacts with the external medium that surrounds it in the acceleration region. It is important, therefore, to consider the effects of the ambient "weather" surrounding the jet.

Highly-magnetized flows are characterized by a high ratio of Alfven to sound speed $c_{s}$

$$
\frac{\dot{\varepsilon}_{\text {fields }}}{\dot{\varepsilon}_{\text {particles }}}=\frac{v_{\text {jet }} B^{2} / 4 \pi}{v_{\text {jet }} \rho c_{s}^{2}} \approx\left(\frac{V_{A}}{c_{s}}\right)^{2}>>1
$$

This means that, early in the flow the velocity becomes supersonic, but continues to accelerate toward the Alfven speed. In this region, where $c_{s}<v_{j e t}<V_{A}$, the ratio of Poynting flux to kinetic energy flux is

$$
\frac{\dot{\varepsilon}_{\text {Poynting }}}{\dot{\varepsilon}_{\text {kinetic }}}=\frac{\frac{c}{8 \pi}|E \times B|}{v_{\text {jet }} \frac{1}{2} \rho v_{\text {jet }}{ }^{2}}=\frac{B^{2}}{4 \pi \rho v_{\text {jet }}{ }^{2}}=\left(\frac{V_{A}}{v_{\text {jet }}}\right)^{2}>1
$$

That is, the flow is "Poynting-flux-dominated" as long as the flow remains supersonic but sub-Alfvenic.

While semi-analytic models of such "Poynting-flux-dominated" jets have been built [Li et al. 1992 Lovelace et al. 2002], no numerical simulations of highly relativistic jets have been performed yet. The best numerical results so far are from non-relativistic simulations [Nakamura et al. 2001, Nakamura \& Meier 2003, which compute the behavior of a jet in a decreasing density (increasing $V_{A}$ ) atmosphere. They show that the electromagnetic power is carried by a fairly coherent torsional Alfven wave that encompasses the jet in a twisting spiral pattern. This wave can transport energy and momentum along the flow, causing further acceleration far from the central engine.

Simulations of both stable and unstable jets are shown in Figure 1.1 We find that the stability of Poynting flux-dominated flows is critically dependent on how severe the mass entrainment in the jet is - specifically on the gradient of the plasma parameter $\beta_{\text {plasma }} \equiv$ $p_{\text {gas }} /\left(B^{2} / 8 \pi\right)$. (In the following, remember that $\beta_{\text {plasma }}$ is always less than unity for PFD jets, if the plasma is reasonably cold $P \leq \rho c^{2}$.) If $\beta_{\text {plasma }}$ decreases or remains small as the jet propagates outward (mass loading becomes even less or stays the same), then we find that the PFD jet remains stable. However, if $\beta_{\text {plasma }}$ increases (entrains significantly more thermal material), then we find that the jet is likely to be unstable to the helical kink instability, even if the jet still remains magnetically dominated throughout the simulation. Apparently even a small amount of pressure in the flow builds up over large distances, triggering a helical kink and, therefore, turbulence in the jet.

Collimated MHD outflows that are strongly dominated by magnetic stresses, and which remain that way or increase in strength, therefore propagate as straight and stable jets. Those in which the relative strength of the field decreases along the jet become unstable to the helical kink instability. In the latter case the outflow decelerates and deposits its momentum in a broad cone rather than punching through the ambient medium in a narrow jet. $\beta_{\text {plasma }}<<1$ 

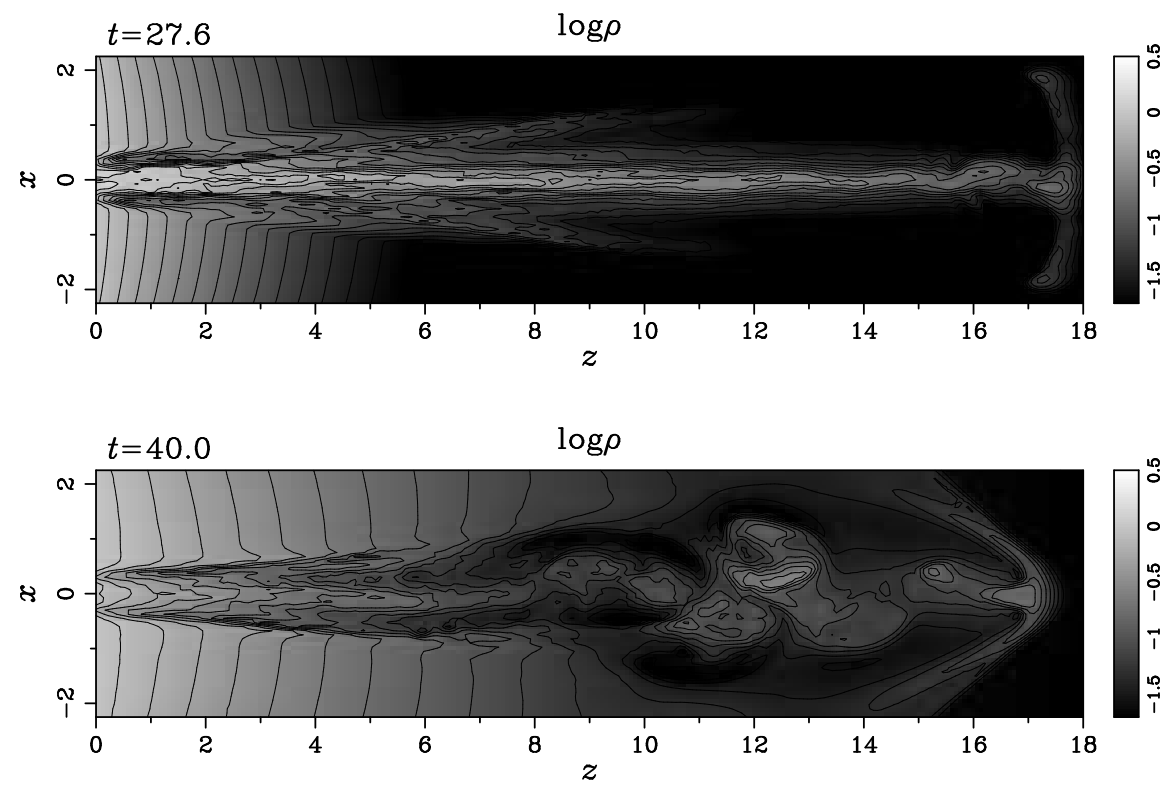

Fig. 1.1. Three-dimensional simulations of jets that are stable (top) and unstable (bottom) to the helical kink instability. Both have $\beta_{\text {plasma }}<1$ throughout the domain, but the stable model has $d \ln \beta_{\text {plasma }} / d \ln r<\sim 0(-d \ln \rho / d \ln r \sim 3, v s$. 2 for the unstable model). (After |Nakamura \& Meier 2003.).

is, therefore, a necessary, but not sufficient condition for jet stability. We also must have $d \ln \beta_{\text {plasma }} / d \ln r<\sim 0$. This may have important implications for MHD outflows from newly-formed pulsars in the centers of core-collapse SNe.

\subsection{MHD-Jet-Powered Supernovae}

\subsubsection{Basic MHD Supernova Model}

Several authors have suggested in the past that MHD phenomena may power supernovae [LeBlanc \& Wilson 1970 Bisnovatyi-Kogan 1971]. The recent discovery that SN ejecta are elongated by an asymmetric jet-like flow has stimulated renewed interest in these models and, in particular, in the possibility that an MHD jet produced by the protopulsar may be the source of the explosion energy in all core-collapse SN. We have proposed Wheeler et al. 2002 an explosion mechanism that is consistent with the above properties of MHD jets. The jet is produced in the iron mantle, just outside the protoneutron star. An object with a $10^{15} \mathrm{G}$ field and a rotation period of $\sim 1 \mathrm{~ms}$ can produce a jet power of $\sim 3 \times 10^{51} \mathrm{erg} \mathrm{s}^{-1}$ and a total energy of $\sim 2 \times 10^{52} \mathrm{erg}-$ more than enough to eject the outer envelope and account for the observed explosion energy. The ejecta from the vicinity of the core is nickel-iron rich, with a mass of $\sim 10^{32} \mathrm{~g}$, an initial velocity of $\sim 0.5 c$, and a total momentum of $\sim 1.5 \times 10^{42} \mathrm{~g} \mathrm{~cm} \mathrm{~s}^{-1}$, decelerating somewhat as it passes through the stellar envelope. 
The protoneutron star spins down to more respectable rotation periods $(>10 \mathrm{~ms})$ in about $\sim 10 B_{15}^{-2}$ seconds. (The model is similar to that of Ostriker \& Gunn [Ostriker \& Gunn 1971, with their $10^{12} \mathrm{G}$ pulsar fields replaced by $10^{15} \mathrm{G}$ protopulsar fields.) The jet outflow is composed of iron-rich material and is initially broad at the base. Furthermore, as even these field strengths do not satisfy the necessary condition for jet stability, the jet likely will be subject to helical kink instabilities, broadening further into a wide bipolar outflow at large distances $\left(>10^{7-8} \mathrm{~cm}\right)$ from the core. It therefore can couple well to the outer envelope and eject it, imparting an elongated shape to the supernova explosion.

\subsubsection{The Pulsar Rocket}

The Crab pulsar currently has a proper motion of $\sim 120 \mathrm{~km} \mathrm{~s}^{-1}$ along the pointing direction of one of its twin jets. This motion has an energy of $\sim 2 \times 10^{47} \mathrm{erg}$ and momentum of $\sim 3 \times 10^{40} \mathrm{~g} \mathrm{~cm} \mathrm{~s}^{-1}$. However, while the current Crab pulsar jets have sufficient energy to have powered this motion $\left(\sim 10^{49} \mathrm{erg}\right.$, if they had operated continuously over the past 950 $\mathrm{yr}$ ), the present Crab jet does not produce enough momentum to accelerate the pulsar to this speed, even if the jet had been continuously and completely one-sided (100\% asymmetric, or $\left.10^{39} \mathrm{~g} \mathrm{~cm} \mathrm{~s}^{-1}\right)$. On the other hand, the MHD jet inferred from the MHD supernova model above would have been quite sufficient to supply both the energy $\left(\sim 10^{52} \mathrm{erg}\right)$ and momentum with only a $2 \%$ jet asymmetry. The fact that the Crab jet and proper motion are aligned, indicates that the current observed Crab pulsar jet (with a speed of $\sim 0.5 c$ ) may be a vestige of the original $\sim 0.5 c$ jet that exploded the supernova and accelerated the pulsar.

\subsubsection{A Gamma-Ray Burst Trigger}

This model also includes a gamma-ray burst trigger. In very rare instances, the field can be dynamically strong in the iron mantle $\left(B>10^{16} \mathrm{G}\right)$, leading to satisfaction of the necessary (but not sufficient) jet stability condition $\beta_{\text {plasma }}<<1$. If the density gradient then is also steep $\left(d \ln \beta_{\text {plasma }} / d \ln r<\sim 0\right)$ then the jet becomes narrow and very fast, coupling poorly to the mantle, punching through the outer envelope [Khokhlov \& Höflich 2001], escaping the star, and producing a heavy iron "lobe" outside it, traveling at a speed of 0.05-0.3 c. Because of the poor coupling to the mantle, the explosion fails, and much of the mantle falls back onto the protoneutron star, putting the system into a state very similar to that at the beginning the "failed SN" GRB model of [MacFadyen \& Woosley 1999]. When the mantle fallback accretes enough material onto the protoneutron star (after several minutes to hours), the neutron star is crushed to a black hole, and a new very fast $\left(\Gamma_{j e t}>>1\right)$ jet is produced via the $\mathrm{BZ}$ or PC mechanisms discussed above. The relativistic jet catches up with the slow, iron-rich lobe at a distance of $d \sim v_{\text {jet }} \tau_{\text {fallback }} \sim 10^{12-13} \mathrm{~cm}$, and the interaction of jet and lobe produces gamma-rays, optical afterglow, and an iron-rich spectrum.

\subsubsection{Unresolved Issues}

The $10^{14-15} \mathrm{G}$ magnetic field strengths needed in SN cores are the real key to the success of the MHD SN model. Magnetars are believed to have surface field strengths of this order, but pulsars typically have fields of order $10^{12-13} \mathrm{G}$. Fields of this strength would have produced a slow supernova explosion lasting several months. The prediction, then, is that fast core-collapse SNe produce magnetars and slow SNe produce pulsars, but there is no observational evidence for (or against) this prediction, neither direct nor statistical. On the other hand, if stronger fields did exist in pulsars at the time they were formed, they must 
have been dissipated either during the SN process or shortly thereafter, but it is not known how that dissipation may have taken place. Secondly, there also may be competing jet mechanisms (neutrino radiation pressure, etc.) which we have not discussed here. Thirdly, while we have suggested a possible SN failure mechanism, much more detailed theoretical work will be needed before we will be able to perform the simulations necessary to test this and other such mechanisms. Finally, there is a problem that needs to be addressed by all SN models. The iron mantle in the progenitor star is very neutron rich. If much of it is ejected (and the $1.4 M_{\odot}$ protoneutron star is left), then the predicted amount of $r$-process material may be much larger than that observed. It is a general problem for all core-collapse $\mathrm{SN}$ models to produce a neutron star remnant while still not over-producing the $r$-process elements.

\subsection{A Grand Unified Scheme for All Galactic Jet Sources}

SN and long-duration GRBs, therefore, potentially can be unified astrophysically as being different possible outcomes in the final stages of the death of a massive star. Also, if the above model is applicable, they both can be unified physically as being powered by MHD jets. This strongly suggests, therefore, a possible grand scheme that unifies all Galactic jet sources discussed in Sect. 1.1 both physically and astrophysically, in a similar manner.

The properties of all Galactic jet sources are similar in several ways. They all appear to produce jets when there is accretion, shrinkage, or collapse of plasma in a gravitational field. Because of that shrinkage or collapse, they occur in systems that are probably in a rapid rotation state: conservation of angular momentum implies that even a modest amount of rotation before the collapse would be amplified greatly. They also are associated with systems that have strong magnetic fields. Some directly reveal these magnetic fields in their radio synchrotron emission. Others are produced by stars that are believed to have strong fields for other reasons (protostars, accretion disks, newly-formed protoneutron stars). The grand unified scheme, therefore, asserts that all Galactic jet sources represent objects in which an excess of angular momentum has built up because of accretion or collapse. And the production of the jet itself represents the expulsion of that excess angular momentum by electro-magneto-hydrodynamic processes. The unifying evolutionary sequence for all Galactic jet sources is shown in Figure 1.2

\subsubsection{Protostellar and White Dwarf Galactic Jets}

The unified sequence begins with the formation of a protostar in a collapsing interstellar cloud. A jet is formed during the accretion phase, and is responsible for spinning down the star to the relatively low rotation rates seen on the main sequence. In low-mass stars jet production does not resume until shrinkage of the central stellar proto-white-dwarf core produces a bipolar planetary nebula outflow. Symbiotic star-type jets are expected in binary systems, but isolated white dwarfs may have neither the accretion fuel nor the rapid spin to do so.

\subsubsection{Neutron Star and Black Hole Jets}

The MHD supernova model discussed earlier provides the missing link to GRB, $\mathrm{X}$-ray binary, and microquasar jets. In the grand unified scheme, most massive star cores collapse to a neutron star, ejecting a broad MHD jet in the process that drives the SN explosion and produces the observed asymmetry. After the envelope dissipates, if the pulsar is 


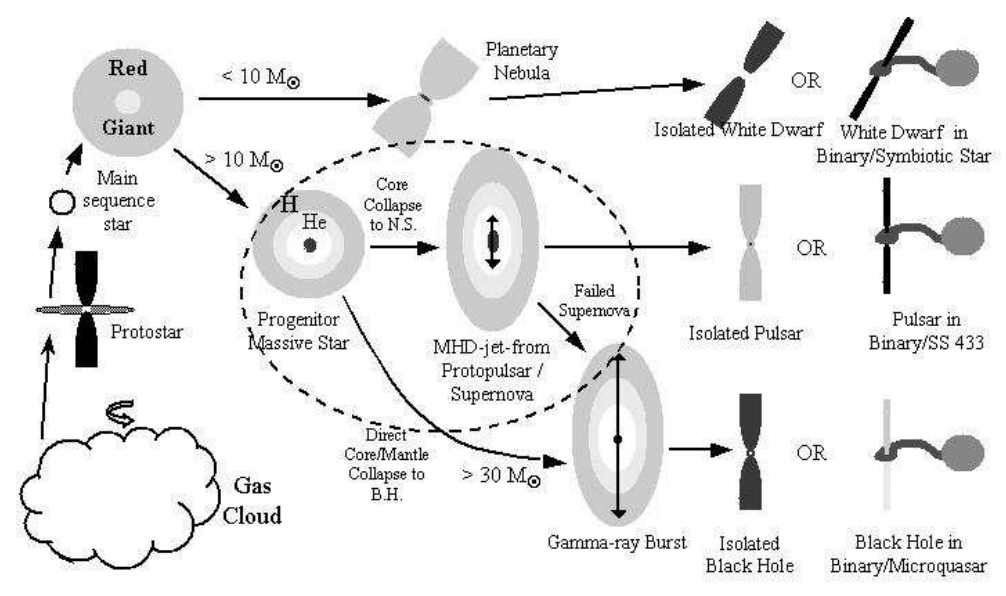

Fig. 1.2. The proposed grand unified model for Galactic jet sources. (After [Meier 2003].)

an isolated object, residual rotation of the magnetized remnant still drives an MHD outflow and a moderately relativistic jet like that seen in the Crab and Vela pulsars. On the other hand, if the pulsar resides in a binary system, it may accrete material from its companion star in a super-Eddington phase $\left(\dot{M}_{a c c}>>10^{18} \mathrm{~g} \mathrm{~s}^{-1}\right)$ and appear like SS433 for a brief time. Cessation of the accretion, angular momentum evolution of the pulsar, or possible collapse to a black hole all could serve to alter SS433's present state.

In rare circumstances, the MHD SN jet will fail to eject the envelope, or perhaps the progenitor core will collapse directly to form a black hole. In either case a GRB event should be generated in a manner similar to that in the failed SN model. The GRB jet event will not spin down the black hole completely (although a significant amount of rotational energy may be extracted from the black hole by another means - gravitational waves). After the envelope dissipates, if the black hole is an isolated object, it may emit little radiation and be difficult to detect. On the other hand, if the newly-formed hole is in a binary system, it also can accrete plasma and field from its companion, thereby producing a strong jet and classical microquasar. Again, changes in the accretion rate and angular momentum evolution of the black hole will alter the microquasar's observational state.

The key element of this unified model is that the evolutionary outcome of a star is ultimately determined by the magnitude and direction of the angular momentum and magnetic field of that star core's. We therefore should consider the jets seen in young pulsars and SS433-type objects to be vestiges of the mechanism that exploded the massive stars from which they came and, in a similar manner, consider the jets in microquasars to be the remnants of the gamma-ray burst that triggered the black hole's formation eons ago. These relatively modest jets are the echoes of violent events of the distant past.

The research described in this paper was carried out at the Jet Propulsion Laboratory, California Institute of Technology, under contract to the National Aeronautics and Space Administration. M.N. was supported, in part, by a National Research Council Resident Research fellowship from NASA. 


\section{References}

Bisnovatyi-Kogan, G., 1971, Soviet Astron. AJ, 14, 652.

Blandford, R., 1976, M.N.R.A.S., 176, 465.

Blandford, R. \& Znajek, R., 1977, M.N.R.A.S., 179, 433 (BZ).

Blandford, R. \& Payne, D., 1982, M.N.R.A.S., 199, 883 (BP).

Junor, W. et al. , 1999, Nature, 401, 891.

Khokhlov, A. \& Höflich, P., 2001, Explosive Phenomena in Astrophysical Compact Objects, AIP, 301.

Koide, S. et al. , 2002, Science, 295, 1688.

Krasnopolsky, R. et al. , 1999, Astroph. J., 526, 631.

Kudoh, T. et al. , 1999, Numerical Astrophysics, Kluwer, 203.

LeBlanc, J. \& Wilson, J., 1970, Astroph. J., 161, 541.

Leonard, D., et al. , 2001, Astroph. J., 553, 861.

Li, Z.-Y. et al. , 1992, Astroph. J., 394, 459.

Lovelace, R., 1976, Nature, 262, 649.

Lovelace, R. et al. , 2002, Astroph. J., 572, 445.

MacFadyen, A. \& Woosley, S., 1999, Astroph. J., 524, 62.

Meier, D., et al. , 2001, Science, 291, 84.

Meier, D.L., 2003, in 4th Microquasar Workshop, Center for Space Physics, Kolkata, India, 165.

Nakamura, M. et al. , 2001, New Astronomy, 6, 61.

Nakamura, M. \& Meier, D.L., 2003, in preparation.

Punsly, B. \& Coroniti, F., 1990, Astroph. J., 354, 583 (PC).

Ostriker, J. \& Gunn, J., 1971, Astroph. J., 164, L95.

Shibata, K. \& Uchida, Y., 1985, Pub. Astron. Soc. Japan, 37, 31.

Sikora, M. \& Madejski, 2001, astro-ph/0112231

N.Vlahakis \& A.Konigl, Astrophys.J., 563, L129 (2001).

Wang, L., et al. , 2001, Astroph. J., 550, 1030.

Wang, L., et al. , 2003, this conference.

Wheeler, J. et al. , 2002, Astroph. J., 568, 807. 